\title{
Effective Diffusion in Flake-Polymer Composites with Accelerated Interphase Transport ${ }^{\dagger}$
}

\author{
by \\ Bryan B. Pajarito ${ }^{* * * *}$, Masatoshi Kubouchi ${ }^{* * *}$, Tetsuya SakaI ${ }^{* * * *}$ and Saiko AokI ${ }^{*}$
}

Flake-polymer composites are commonly used in protection and barrier applications due to its low effective diffusivity. Effective diffusivity through flake-polymer composites depends on the local accelerated transport along the flakematrix interphase. This paper describes analytical models for predicting the effective diffusivity through the flake-polymer composite as a function of flake orientation, volume fraction, aspect ratio, and interphase properties. Estimates of effective diffusivity were also generated using random walk simulations. Results from the two methods agree well with each other. Using model-predicted effective diffusivities, solute penetration depths in systems with flakes aligned either orthogonal or parallel to the diffusion direction are calculated and compared to experimentally obtained penetration depths of acid solutes through glass flake-polymer composites.

Key words : Flake-polymer composites, Diffusion, Interphase, Modelling, Random walk simulation

\section{Introduction}

Flake-polymer composites have been an important class of materials popularly used on planar structures due to their capability of reinforcement in two dimensions. ${ }^{1)}$ Moreover, since the flakes are impermeable, flake-polymer composites also find application as protective coatings and anti-corrosion linings. A critical aspect of using flake-polymer composite materials in barrier applications is their effective diffusivity due to flake reinforcement. Impermeable flakes reduce the effective diffusivity of the composite by decreasing the area available for diffusion and increasing the effective path length of diffusing species. ${ }^{2}$

A lot of analytical/numerical diffusion models, taking into consideration several parameters such as flake aspect ratio, volume fraction, spacing, orientation, random dispersion, size distribution, and filler shape, are proposed and studied in literature for prediction of effective diffusion in flake-filled media. ${ }^{2) ~ 9)}$ However, these models only predict effective diffusion in a flake-polymer composite with perfect interphase bonding. The interphase is a special region in the vicinity of the dispersed flakes with properties and response different from that of the polymer matrix. Recent experimental work has reported accelerated local transport of sulfuric acid solute at glass flake-epoxy interphase. ${ }^{10)}{ }^{11)}$ In this case, the solute penetration was $3-4$ times longer in the interphase than in the bulk epoxy matrix. Moreover, based from specimen images taken by scanning electron microscopy (SEM) coupled with energy dispersed spectroscopy (EDS) for sulfur element detection, the interphase regions are thicker compared to dispersed glass flakes. As a result, measured effective diffusivities and solute penetration depths from this work deviate largely from the published diffusion models.

The present work complements and explains the previous experimental results by proposing models that predict effective diffusivity in flake-polymer composites with accelerated interphase transport. Effective diffusivities are also estimated using random walk computer simulations. Aside from the interphase, the effect of flake orientation, aspect ratio, and loading are discussed. The results of the model are compared to the experimental results previously reported ${ }^{10) \sim 12)}$ in terms of solute penetration depth.

\section{Theory}

\section{1 Diffusion Orthogonal to Flakes}

The derivation procedure presented here follows the approach of Cussler et al. ${ }^{9)}$ Figure 1 shows the idealized model of flake network in the composite. The flakes are

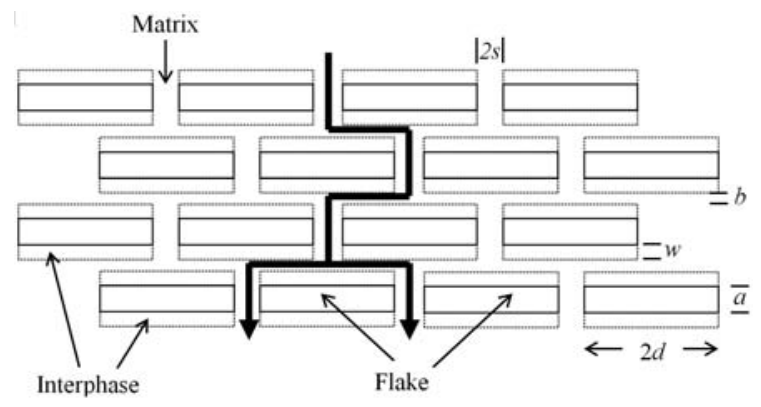

Fig. 1 Model of diffusion in flake-polymer composites. 
assumed to be rectangles of uniform size and regularly spaced. Each flake is bounded by two interphase regions of uniform thickness. In this case, diffusion will occur through the slits between the flakes and through the interphase regions.

Considering a unit cell of area $(2 d W)$, where $W$ is the flake depth normal to model plane, $2 d$ is the flake width, and $2 s$ is the slit size such that $d \gg s$ in Fig. 1, the total flux $J_{o}$ through the unit cell without flakes is :

$$
J_{o}=\frac{D(2 d W)}{l} \Delta C
$$

where $l$ is the total thickness of the composite and $\Delta C$ is the concentration difference across it. The diffusion resistance is then written as :

$$
\frac{D \Delta C}{J_{o}}=\frac{l}{2 d W}
$$

which is proportional to composite thickness and inversely proportional to the area normal to diffusion.

At the presence of flakes arranged in Fig. 1, a diffusing solute will pass on a slit and then hit a flake. It can then either diffuse to the left or to the right. During this time, the diffusion resistance is due to parallel contributions of matrix and interphase regions. Hence, the parallel diffusion resistance due to matrix and interphase after hitting a flake is :

$$
\begin{aligned}
\frac{1}{R_{m+i}} & =\frac{1}{R_{i}}+\frac{1}{R_{m}}+\frac{1}{R_{i}} \\
& =\frac{2 D_{i} w W}{d}+\frac{2 D b W}{d}+\frac{2 D_{i} w W}{d} \\
R_{m+i} & =\frac{d}{2 D W} \cdot \frac{1}{2 \beta w+b}
\end{aligned}
$$

in which $R$ is the diffusion resistance ; $D_{i}$ and $D$ are the diffusivities of interphase region $i$ and matrix $m ; b$ is the flake layer spacing ; $w$ is the interphase thickness ; and $\beta$ is the ratio $\left(D_{i} / D\right)$. On the other hand, the diffusion resistance due to the presence of slits $R_{\text {slit }}$ is :

$$
R_{\text {slit }}=\frac{a}{2 s D W}
$$

where $a$ is the flake thickness. Using Eqs. (2), (3) and (4), the total diffusion resistance for a flake-polymer composite containing $N$ layers of flakes is given by :

$$
\frac{D \Delta C}{J_{N}}=\frac{l}{2 d W}+N \frac{a}{2 s W}+(N-1) \frac{d}{2 W} \cdot \frac{1}{2 \beta w+b}
$$

in which $J_{N}$ is the total flux through the unit cell containing $N$ layers of flakes. The first term on the right-hand side of Eq. (5) is the resistance without flakes, just as in Eq. (2). The second term is the resistance of $N$ slits through which the solute must pass to cross the composite, which is taken from Eq. (4). The last term represents the $(N-1)$ flakes the solute will hit after passing the slit and reflects the parallel resistance due to matrix and interphase regions, as in Eq. (3).
Dividing Eq. (2) with Eq. (5) will give the flux ratio :

$$
\frac{J_{N}}{J_{o}}=\frac{1}{1+N \frac{a d}{l s}+(N-1) \frac{d^{2}}{l(2 \beta w+b)}}
$$

For a flake-polymer composite with many layers of flakes, $N,(N-1)$, and $(N+1)$ are almost the same. Also in Fig. 1 , the composite thickness $l$ is equal to $N(a+2 w+b)$. If each layer is almost filled with impermeable flakes, the flake volume fraction $\phi$ equals $a d /(a+2 w+b)(d+s) \approx(a /(a+$ $2 w+b)$ when $d \gg s$. Three new variables are also defined. The flake aspect ratio $\alpha(=d / a)$ is a measure of flake shape, while the slit shape factor $\sigma(=s / a)$ characterizes the slit size. The interphase volume fraction $\phi_{i}$ is $2 w d /(a+$ $2 w+b)(d+s) \approx(2 w /(a+2 w+b)$. With these changes, Eq. (6) becomes

$$
\begin{aligned}
\frac{J_{N}}{J_{o}}=\frac{D_{\perp}}{D} & =\frac{1}{1+\frac{a d}{s(a+2 w+b)}+\frac{d^{2}}{(a+2 w+b)(2 \beta w+b)}} \\
\frac{D_{\perp}}{D} & =\frac{1}{1+\frac{\alpha \phi}{\sigma}+\frac{\alpha^{2} \phi^{2}}{1-\phi+\phi_{i}(\beta-1)}}
\end{aligned}
$$

where $D_{\perp}$ is the effective diffusivity of the flake-polymer composite when flakes are oriented orthogonal to diffusion direction. Equation (7) is similar to the earlier diffusion model published by Cussler et al. ${ }^{9)}$ except for the expression $\phi_{i}(\beta-1)$ which takes into account the accelerated local interphase transport.

A simplified case is observed when composites are reinforced with flakes of high flake size such that $\sigma / \alpha \gg 1{ }^{9}$ ) making the effects of slits negligible. Moreover, if the flakes are randomly dispersed, Eq. (7) is now generalized as :

$$
\frac{D_{\perp}}{D}=\frac{1}{1+\mu \frac{\alpha^{2} \phi^{2}}{1-\phi+\phi_{i}(\beta-1)}}
$$

Using a combined geometric factor $\mu$ ( $\mu=1$ for the flake arrangement in Fig. 1 and 0.5 for random flakes ${ }^{2)}$ ) for randomly distributed $2 D$ flakes, Eq. (8) proposes effective diffusion in orthogonal flakes to be dominated by tortuosity and accelerated interphase transport. Both Eqs. (7) and (8) have application in the "semi-dilute" limit, where $\alpha \phi>1$ and $\phi \ll 1$. In this limit, the flake concentration is small but the flakes overlap which contributes to increased tortuosity. On the other hand, the expression $\phi_{i}(\beta-1)$ where $\phi_{i}>0$ and $\beta>1$ represents the interphase regions which are diffusion routes for accelerated transport. Fig. 2 shows the predictions of Eqs. (7) and (8) compared with the conventional diffusion models proposed by Cussler et al. ${ }^{9)}$ for staggered and random flake networks. Though similar in trend with the conventional diffusion models, the estimates of Eqs. (7) and (8) are higher due to accelerated interphase transport. 


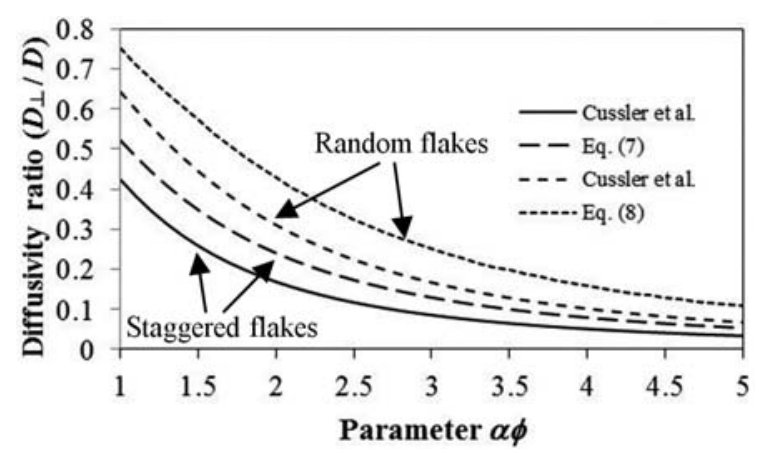

Fig. 2 Comparison of predictions of Eqs. (7) and (8) with conventional diffusion models by Cussler et al. ${ }^{9)}$ Parameters are set at $\phi=0.1, \sigma=4, \mu=0.5, \phi_{i}=$ 0.2 , and $\beta=4$.

\section{2 Diffusion Parallel to Flakes}

By linear mixing rule, the effective diffusivity of composite when flakes are parallel to diffusion direction, $D_{\|}$:

$$
D_{\|}=\phi D_{f}+\phi_{m} D+\phi_{i} D_{i}
$$

Setting diffusivity of impermeable flake $D_{f}$ to zero and matrix volume fraction $\phi_{m}$ to $\left(1-\phi-\phi_{i}\right)$ :

$$
\frac{D_{\|}}{D}=1-\phi+\phi_{i}(\beta-1)
$$

Equation (10) suggests effective diffusion in parallel flakes to be enhanced with increasing interphase volume fraction and deceasing volume fraction of impermeable flakes.

\section{Numerical}

The validity of Eqs. (7) and (10) were verified using a computer simulation of random walks ${ }^{13)}$ with step size modification to simulate accelerated interphase transport. Simulations were executed by a random walking particle in a model flake network shown in Fig. 3. A particle is allowed to randomly walk through the network on the space unoccupied by obstructing flakes. At the start

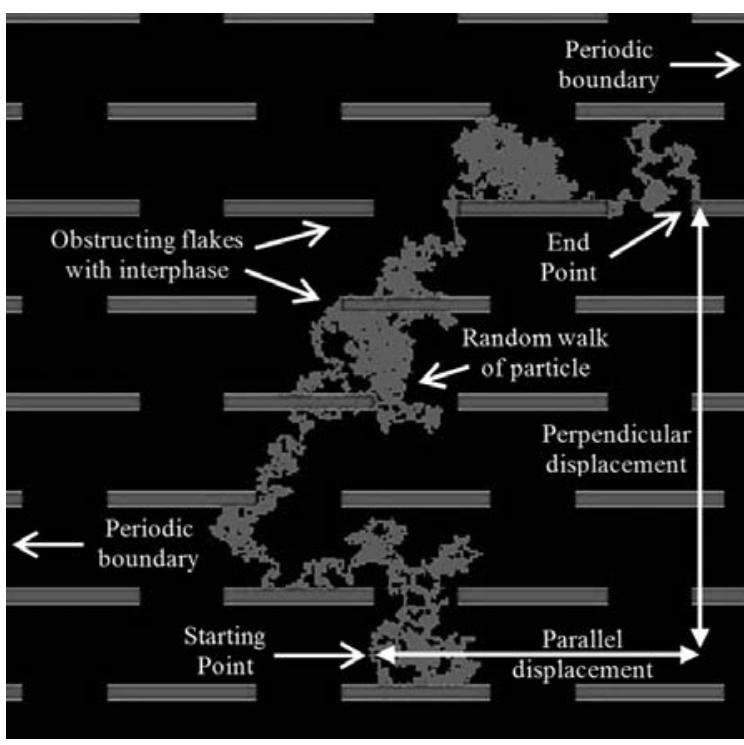

Fig. 3 Schematic diagram showing random walk process. of each simulation, flakes of specified sizes and shapes are placed in the matrix as shown in Fig. 3. A particle is then randomly placed on an empty site in the matrix and the site is assigned as the starting point. The particle is then allowed to move in any direction : up, down, left, or right, with equal probability. The direction of movement is chosen based on a number generated by the random number generator between 1 and 4 . For example, if random number generated is 1 , then up direction is considered. If the position on the chosen direction is flake-free, the particle will move to the new position ; otherwise another random number is called and the process is repeated. Each call to the random number generator is counted as a time step. Periodic boundary conditions are imposed as shown in Fig. 3 to make the simulation medium repeating. After a specified number of particle steps, the process is stopped and the end point of particle is located. Perpendicular and parallel displacements, $z_{\perp}$ and $z_{\|}$, are then calculated as shown. The entire procedure is repeated for many particles and the mean square displacement $\left\langle z^{2}\right\rangle$ is computed. If $\left.<z_{0}^{2}\right\rangle$ is the mean square displacement computed in a flake-free matrix at the same number of particle steps, then the mean square displacement ratio $\left.\left(<z^{2}>/<z_{0}^{2}\right\rangle\right)$ is equal to the diffusivity ratio $\left(D_{\perp} / D\right)$ or $\left(D_{\|} / D\right)$, depending on the direction of measured displacement. ${ }^{11) \sim 13)}$

To simulate accelerated interphase transport, a particle is allowed to move larger steps when situated at the interphase regions as illustrated in Fig. 4. Large particle step is only considered at the interphase region if the random generated direction is parallel to the flake. Fig. 5 shows the flowchart of the random walk simulation used in this study.

Appropriate number of particle steps and number of particles are used during the simulation to ensure consistent results with minimum deviation. Varied values of $d(60,30), a(5,3)$, and $2 s=b(26,30,34)$ are arbitrarily chosen during computer generation of flake network for random walk simulation. The interphase thickness $w$ is set to 1 .

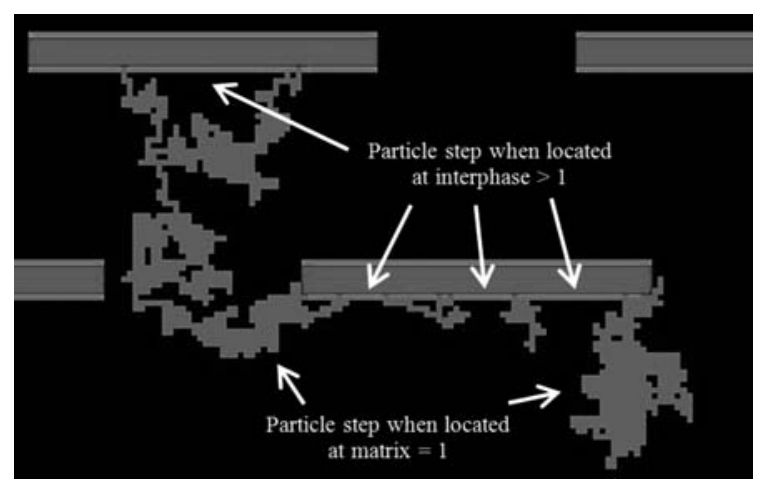

Fig. 4 Particle step at interphase during random walk. 


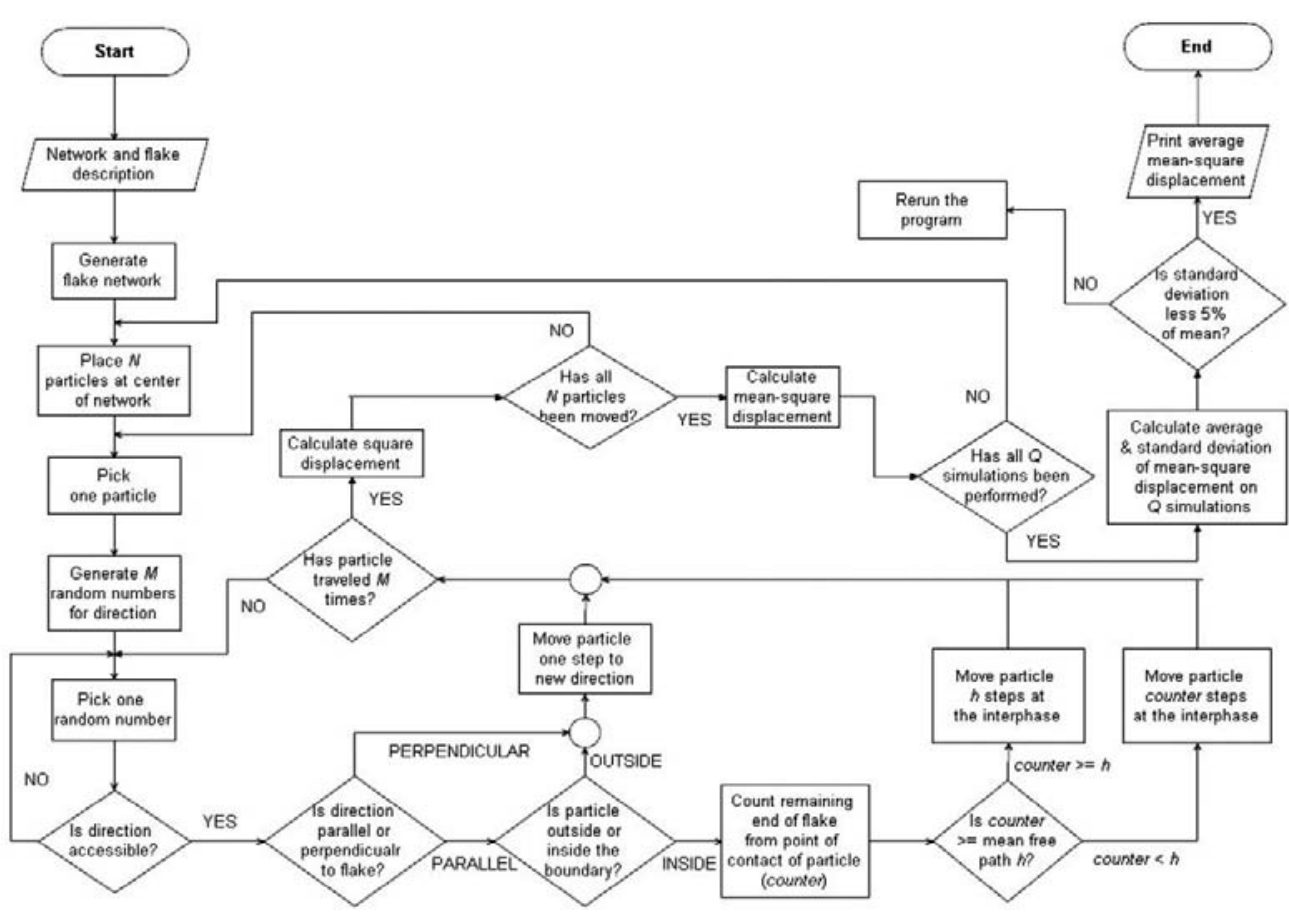

Fig. 5 Flowchart of random walk simulation with accelerated local interphase transport in flake-filled network. A particle situated in the interphase region moves faster than a particle situated in the bulk matrix.

\section{Results and Discussion}

Figure 6 shows results for the effective diffusivity ratio $\left(D_{\perp} / D\right)$ obtained using random walk simulations for flakes for various aspect ratios $\alpha(=d / a=20,12,10$ and 6$)$ and slit shape factors $\sigma(=s / a)$. The results are also shown for varied interphase particle step size $h(1,2$ and 3$)$. The particle step size $h$ is synonymous to the mean free path which is the average distance a diffusing molecule travels between collisions with other moving molecules. Since experimental diffusion of acid solution is observed to be accelerated $3-4$ times at the flake-polymer interphase regions compared to the polymer matrix, a high particle step size $h$ is assigned at the interphase during random walk simulation. It can be seen that the effective diffusivity $D_{\perp}$, when flakes are aligned normal to diffusion direction, increases with decreasing aspect ratio and slit shape factor. As the flakes become smaller and the slits grow larger,

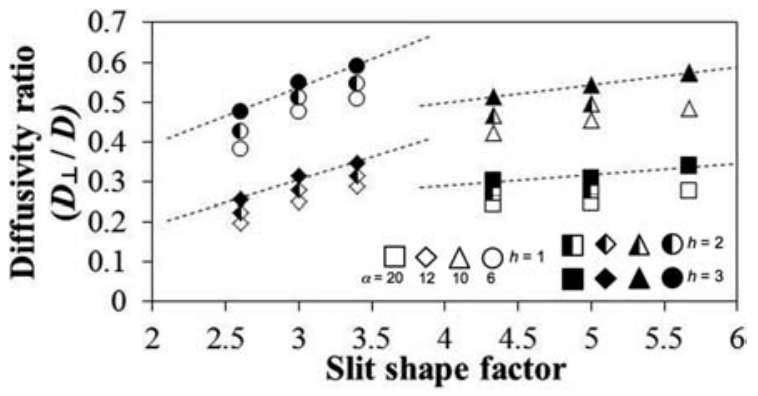

Fig. 6 Random walk simulation results for perpendicular flakes at varied aspect ratios $\alpha$ and interphase particle step size $h$. the path through the flake network becomes less tortuous, leading to a larger effective diffusion coefficient. The effective diffusivity also increases as the interphase particle step size becomes larger.

Figure 7 shows results for the effective diffusivity ratio $\left(D_{\|} / \mathrm{D}\right)$ using random walk simulations for parallel flakes. Unlike the result for $\left(D_{\perp} / \mathrm{D}\right)$ when flakes are perpendicular to diffusion direction, the effective diffusivity $D_{\|}$increases with increasing aspect ratio and decreasing slit shape factor. Moreover, the effective diffusivity greatly increases with interphase particle step. As the flakes become larger and the slits become smaller, the chance for the particle to move perpendicular to flakes grows less, resulting in a larger effective diffusivity. This effect is more pronounced as the interphase particle step is increased. The flakes no longer act as obstructions ; instead they serve as conduits for easy transport due to the presence of highly diffusive flake-matrix interphase regions.

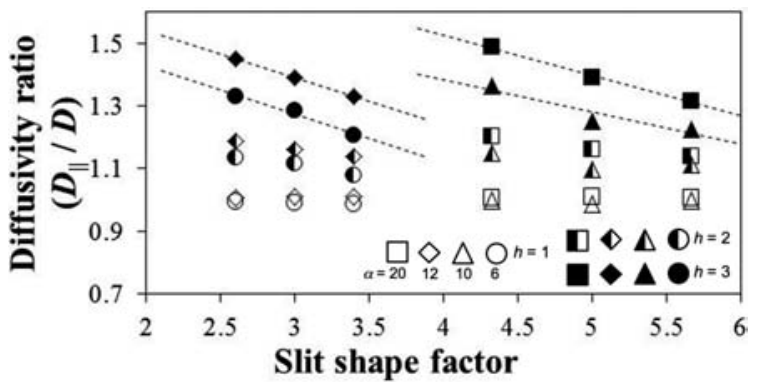

Fig. 7 Random walk simulation results for parallel flakes at varied aspect ratios $\alpha$ and interphase particle step size $h$. 


\section{1 Model Comparisons}

Figure 8 compares results of effective diffusivity ratio $\left(D_{\perp} / \mathrm{D}\right)$ obtained using both Eq. (7) for perpendicular flakes and random walk simulations. The volume fraction of flakes at various aspect ratios and slit shape factors can be calculated from values of $d, a$, and $s$ by using $\phi=a d /$ $(a+2 w+b)(d+s)$, and the volume fraction of interphase regions by $\phi_{i}=2 w d /(a+2 w+b)(d+s)$ for use in Eq. (7). The predicted effects of aspect ratio, slit shape factor, volume fraction, and interphase properties are similar for both approaches, and the two models agree very well. However, deviations are observed at low aspect ratios, and become more pronounced as slit shape factor and interphase particle decrease. This aspect ratio-dependent discrepancy is attributed to the assumption used on the development of Eq. (7). Similar to the diffusion model published by Cussler et. al. ${ }^{9}$ ) without the interphase effects, Eq. (7) is applicable if the flakes are in the "semi-dilute" regime, ${ }^{6)}$, 7) specifically characterized by the conditions: $\alpha \phi>1$ and $\phi \ll 1$. In this concentration regime, flakes are far enough from each other to assume negligible flake-to-flake interaction, yet large enough in size to contribute on diffusion resistance. The lower aspect ratios used in the simulation failed to satisfy this criterion, resulting in a lower effective diffusivity compared to Eq. (7).

Similarly, Fig. 9 compares the effective diffusivity ratio $\left(D_{\|} / D\right)$ computed using both Eq. (10) for parallel flakes and random walk simulations. The estimated effects of volume fraction and interphase properties are comparable for both methods.

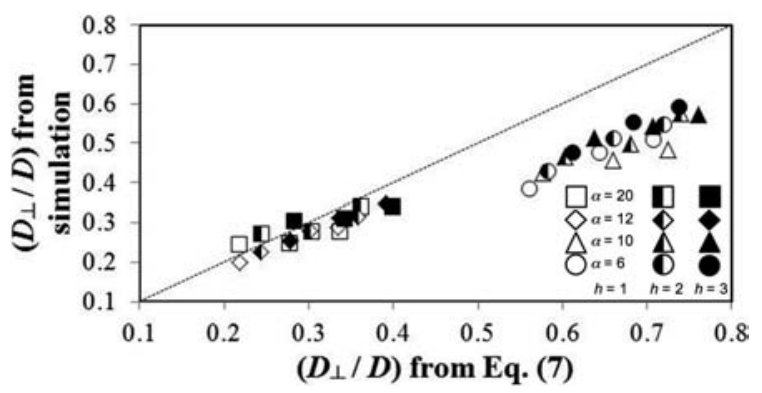

Fig. 8 Comparison of results obtained from Eq. (7) and random walk simulations for perpendicular flakes.

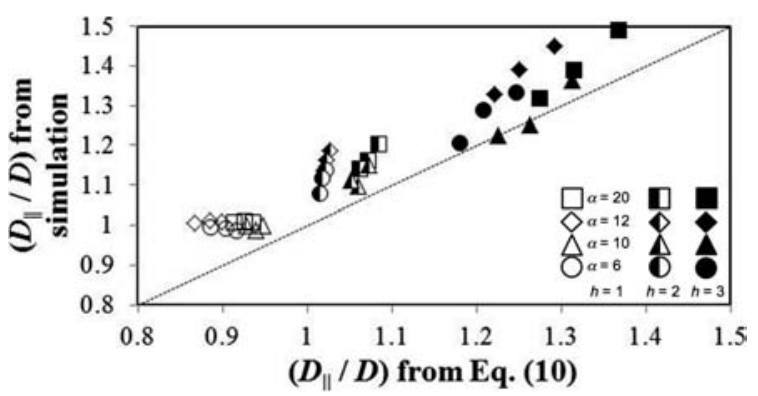

Fig. 9 Comparison of results obtained from Eq. (10) and random walk simulations for parallel flakes.

\subsection{Application to Flake-Polymer Composites}

Solute penetration depths of acids on glass flake-polymer composites were recorded previously. ${ }^{10) \sim 12)}$ Glass flakes were used as reinforcement for amine-cured epoxy and vinyl ester resins. The glass flakes are oriented parallel or perpendicular to the wide surface area of rectangular specimens $(60 \times 25 \times 3 \mathrm{~mm})$. Epoxy composites were immersed in 10 mass\% sulfuric acid solution and vinyl ester composites in 10 mass\% chloroacetic acid solution, both maintained at $80^{\circ} \mathrm{C}$. At specific immersion time, specimens were removed from immersion environment to take the solute concentration profile by SEM-EDS, as shown in Fig. 10. From there, the solute penetration depth on the composite was measured from the surface in contact with solution.

In order to apply the proposed diffusion model for estimation of solute penetration depth in flake-polymer composite, the relationship of penetration depth to diffusion coefficient must first be established. Consider the solute concentration profile in Fig. 10. During early immersion, the penetration of acid solution in composite can be assumed to be diffusion in a semi-infinite medium, where the surface in contact with diffusing environment is kept at constant concentration $C_{0}$. If solute concentration is zero before immersion, the given Fickian problem has the solution $^{14)}$ :

$$
C(x, t)=C_{o} \operatorname{erfc}\left(\frac{x}{2 \sqrt{D_{e} t}}\right)
$$

in which $C$ is the solute concentration at a given immersion time $t$ and distance from surface in contact with diffusing environment $x$ and $D_{e}$ is the effective diffusivity of flake-polymer composite. Since the solute penetration depth $x_{s}$ is measured at $C=0$, then :

$$
\begin{aligned}
& 0=\operatorname{erfc}\left(\frac{x_{s}}{2 \sqrt{D_{e} t}}\right) \\
& \frac{x_{s}}{2 \sqrt{D_{e} t}} \geq 2 \\
& x_{s} \approx 4 \sqrt{D_{e} t}
\end{aligned}
$$

If $x_{o}$ is the solute penetration depth in polymer resin without flakes, then the following is valid ${ }^{10), 12)}$ :

$$
\frac{x_{s}}{x_{o}}=\sqrt{\frac{D_{e}}{D}}
$$

Equation (13) suggests that the distance travelled by solute in the composite is directly proportional to the root of its effective diffusivity ratio. If $x_{0}$ is approximately linear with square root immersion time $t$, such that $x_{o}=m \sqrt{t}$ where $m$ is the slope as observed previously, ${ }^{10), 12)}$ then according to Eq. (13), the solute penetration depths in glass flakes-polymer composites are given as : 


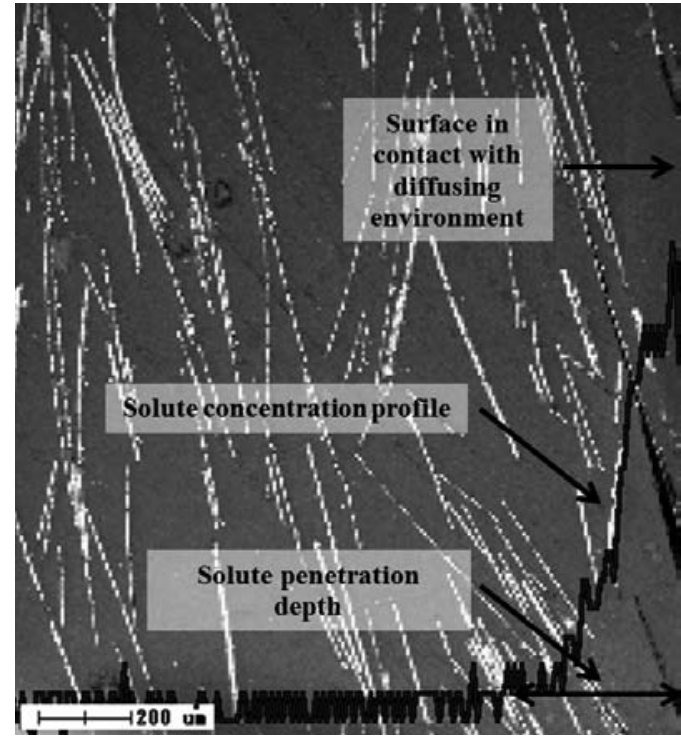

Fig. 10 Measurement of solute penetration depth.

$$
\begin{gathered}
x_{\perp}=\sqrt{\frac{1}{1+\mu \frac{\alpha^{2} \phi^{2}}{1-\phi+\phi_{i}(\beta-1)}} \cdot m \sqrt{t}} \\
x_{\|}=\sqrt{1-\phi+\phi_{i}(\beta-1)} \cdot m \sqrt{t}
\end{gathered}
$$

where $x_{\perp}$ and $x_{\|}$are the solute penetration depths in polymer composites reinforced with perpendicular and parallel glass flakes, respectively.

Figures 11 and 12 compare the experimental solute penetration depth in glass flake-polymer composites with values obtained by Eqs. (14) and (15) at different interphase volume fractions $\phi_{i}$. The diagonal dashed line in the plot runs through the origin with a unit slope for visual comparison of agreement between experimental and predicted results. The solid lines group and distinguish the data points of perpendicular flakes from parallel flakes. Table 1 lists the parameters used in Eqs. (14) and (15) derived from previous experimental results. ${ }^{10), 12)}$ As mentioned earlier, $m$ is the slope of regressed line on a plot of penetration depth $x_{o}$ versus square root immersion

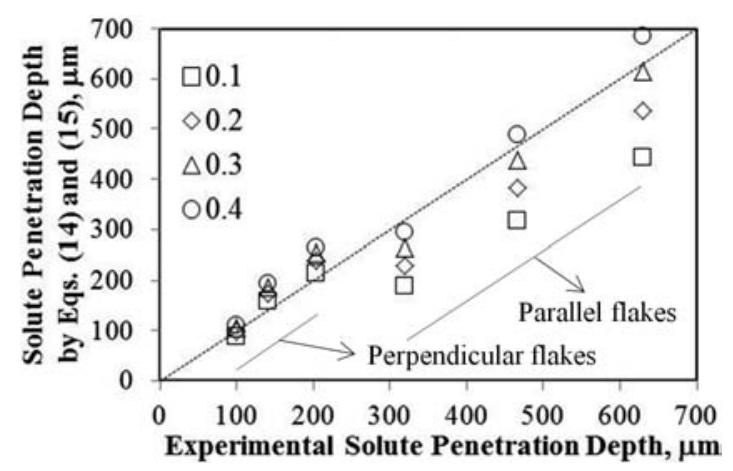

Fig. 11 Comparison of experimental solute penetration depths with Eqs. (14) and (15) at various interphase volume fractions for epoxy composites immersed in 10 mass\% sulfuric acid at $80^{\circ} \mathrm{C}$.

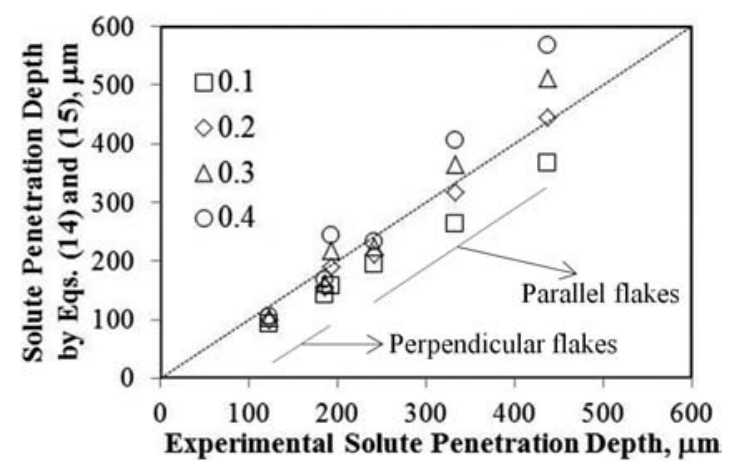

Fig. 12 Comparisons of experimental solute penetration depths with Eqs. (14) and (15) at various interphase volume fractions for vinyl ester composites immersed in 10 mass\% chloroacetic acid at $80^{\circ} \mathrm{C}$.

time $\sqrt{t}$ for neat resins. The value of $m$ for epoxy-sulfuric acid system is $48.19 \mu \mathrm{m} \mathrm{h}^{-0.5}$ and $39.99 \mu \mathrm{m} \mathrm{h}^{-0.5}$ for vinyl ester-chloroacetic acid system. ${ }^{10)}{ }^{12)}$ The geometric factor $\mu$ is assumed as 0.5 for randomly dispersed flakes. ${ }^{2)}$ The volume fraction of flakes $\phi$ was measured by burn-off method, which is 0.076 for epoxy composites and 0.072 for vinyl ester composites. By knowing that the solute penetration at the interphase is 3 times longer than at the bulk matrix for these composites. ${ }^{10) \sim 12)}$ and by virtue of Eq. (13), the interphase diffusivity ratio $\beta$ is set to 9 . The glass flake aspect ratios were approximated by image analysis of SEM images of composite specimen's cross-sections.

With interphase volume fraction $\phi_{i}$ as the only fitting parameter, the magnitudes of solute penetration depths in glass flake-polymer composites are predicted and show good agreement with the experimental results. Small changes in predicted solute penetration depths as function of interphase volume fraction $\phi_{i}$ are observed when the flakes are oriented perpendicular to diffusion, while large changes are shown when the flakes are oriented parallel to diffusion. An interphase volume fraction $\phi_{i}=0.4$ both for perpendicular and parallel flakes are observed for epoxy composite specimens immersed in 10 mass\% sulfuric acid solution, while $\phi_{i}=0.2$ for the vinyl ester composite specimens immersed in 10 mass\% chloroacetic acid. Two competing mechanisms are observed during solute penetration in flake-polymer composites: tortuosity or the blocking effect of flakes, and accelerated interphase transport. When flakes are oriented perpendicular to diffusion, tortuosity dominates the overall penetration of solute in the composite. As flakes become tipped parallel to diffusion, the blocking effect of flakes gets minimized and the solute prefers to penetrate more at the weak interphase regions rather than at the bulk matrix. Also, since the interphase volume fraction is higher than the volume fraction of flakes, the interphase regions are thicker than the glass flakes. Regardless of flake orientation, 
Table 1 Specimens for solute penetration depth measurement.

\begin{tabular}{|c|c|c|c|}
\hline Material & $\begin{array}{c}\text { Flake } \\
\text { Orientation } \\
\text { Relative To } \\
\text { Diffusion } \\
\end{array}$ & $\begin{array}{c}\text { Root } \\
\text { Immersion } \\
\text { Time } \sqrt{t} \\
\mathrm{~h}^{0.5}\end{array}$ & $\begin{array}{c}\text { Flake Aspect } \\
\text { Ratio } \alpha\end{array}$ \\
\hline \multirow{6}{*}{ Epoxy } & \multirow{3}{*}{ Perpendicular } & 3 & 31.3 \\
\hline & & 5 & 27.9 \\
\hline & & 7 & 29.5 \\
\hline & \multirow{3}{*}{ Parallel } & 3 & 26.3 \\
\hline & & 5 & 29.7 \\
\hline & & 7 & 23.9 \\
\hline \multirow{6}{*}{$\begin{array}{l}\text { Vinyl } \\
\text { Ester }\end{array}$} & \multirow{3}{*}{ Perpendicular } & 3 & 21.2 \\
\hline & & 5 & 25.7 \\
\hline & & 7 & 26.8 \\
\hline & \multirow{3}{*}{ Parallel } & 3 & 25.3 \\
\hline & & 5 & 22.9 \\
\hline & & 7 & 23.1 \\
\hline
\end{tabular}

it should be noted that a large extent of interphase and large number of flakes has the possibility of the interphase region to penetrate through the flake-polymer composite and form a percolating network which will provide an easy diffusional route for the penetrating solute.

\section{Conclusions}

In summary, analytical diffusion models based on flake orientation, volume fraction, aspect ratio, and interphase properties for predicting variations in flake-polymer composite effective diffusivity were developed. Varying the mentioned parameters affects the tortuosity and preferred diffusional path and therefore, the effective diffusivity.

Results predicted by the proposed models agree well with the results simulated by the random walk process. Deviations between the analytical models and the random walk simulation are observed in composites with low flake aspect ratios. This is due to the limitation of the analytical model being applicable only to the semi-dilute regime, where flakes of large aspect ratio are preferred. Model and simulation results show that the effective diffusion coefficient of composite increases with increasing local diffusivity at the interphase regions.

Changes in solute penetration depth in glass flake-polymer composites were predicted and compared to experimental penetration depth results. The result indicates that solute penetration depth ratio in composites is directly proportional to the square root of its effective diffusivity ratio. The predicted results agree well with the experimental results, verifying the validity of the assumed mechanism of diffusion in flake-polymer composites with interphase transport.

\section{Acknowledgements}

The authors are grateful to The Hitachi Scholarship Foundation for support to this work.

\section{References}

1) T. Vu-Khanh and B. Fisa, "Impact fracture of glass-flake reinforced polypropylene", Polymer Composites, Vol.7, pp.375-382 (1986).

2 ) G. D. Moggridge, N. K. Lape, C. Yang and E. L. Cussler, "Barrier films using flakes and reactive additives", Progress in Organic Coatings, Vol.46, pp.231-240 (2003).

3 ) J. D. White and E. L. Cussler, "Anisotropic transport in water-swollen flake-filled membranes", Journal of Membrane Science, Vol.278, pp.225-231 (2006).

4 ) D. M. Eitzman, R. R. Melkote and E. L. Cussler, "Barrier membranes with tipped impermeable flakes", American Institute of Chemical Engineering Journal, Vol.42, No.1, pp.2-9 (1996)

5 ) C. Yang, W. H. Smyrl and E. L. Cussler, "Flake alignment in composite coatings", Journal of Membrane Science, Vol.231, pp.1-12 (2004).

6 ) J. P. DeRocher, B. T. Gettelfinger, J. Wang, E. E. Nuxoll and E. L. Cussler, "Barrier membranes with different sizes of aligned flakes", Journal of Membrane Science, Vol.254, pp.21-30 (2005).

7 ) G. H. Fredrickson and J. Bicerano, "Barrier properties of oriented disk composites”, Journal of Chemical Physics, Vol.110, No.4, pp.2181-2188 (1999).

$8)$ N. K. Lape, E. E. Nuxoll and E. L. Cussler, "Polydisperse flakes in barrier films", Journal of Membrane Science, Vol.236, pp.29-37 (2004).

$9)$ E. L. Cussler, S. E. Hughes, W. J. Ward and R. Aris, "Barrier membranes”, Journal of Membrane Science, Vol.38, pp.161 174 (1988).

10) B. B. Pajarito, M. Kubouchi, H. Tomita and T. Sakai, "Degradation behavior of epoxy with tipped glass flakes in sulfuric acid environment”, Proceedings of International Conference on Structural Analysis of Advanced Materials, p.22 (2009).

11) B. B. Pajarito, M. Kubouchi, H. Tomita, S. Aoki and T. Sakai, "Barrier properties of reinforced linings : Effect of polymer-flake interphase", Proceedings of International Symposium on Engineering, Energy and Environment, pp.164-168 (2009).

12) B. B. Pajarito, M. Kubouchi, H. Tomita, S. Aoki and T. Sakai, "Barrier properties of reinforced linings : Effect of flake orientation", Proceedings of 15th Asian-Pacific Corrosion Control Conference, p.145 (2009).

13) Y. P. Ly and Y. Cheng, "Diffusion in heterogeneous media containing impermeable domains arranged in parallel arrays of variable orientation”, Journal of Membrane Science, Vol.133, pp.207-215 (1997).

14) J. Crank, "The mathematics of diffusion", pp.32-35 (1975) Clarendon Press, Oxford, United Kingdom. 